\title{
Defining successful treatment of neurogenic orthostatic hypotension with droxidopa in a patient with multiple system atrophy
}

\author{
Brent P. Goodman ${ }^{1} \cdot$ Fiona Gupta ${ }^{2}$
}

Received: 7 June 2017/ Accepted: 7 June 2017/Published online: 19 June 2017

(c) The Author(s) 2017. This article is an open access publication

Keywords Multiple system atrophy $\cdot$ Neurogenic orthostatic hypotension

\section{Challenge question}

When using droxidopa, what are the criteria to adjust the dosage, combine it with other medications, and define therapeutic success?

\section{Case presentation}

Mrs. M is an 80-year-old woman with the parkinsonian variant of multiple system atrophy (MSA-P). She initially presented with a 3-year progressive history of imbalance and a slow gait. She reported frequent falls, a decline in the ability to complete activities of daily living (ADLs), and noted some uncontrollable jerkiness of her arms (not tremors). She later developed facial dyskinesia shortly after starting treatment with levodopa. She has been ambulatory with a walker and lives in an assisted living facility. Her past medical history is significant for hyperlipidemia, urinary urgency, occasional incontinence, and constipation. Her medications included atorvastatin $10 \mathrm{mg}$ taken orally once daily (QD), oxybutynin $5 \mathrm{mg}$ twice daily (BID), and docusate sodium $100 \mathrm{mg}$ BID.

Approximately 6 months ago, Mrs. M had several episodes of syncope, all occurring when she was rising from a seated to

Brent P. Goodman

goodman.brent@mayo.edu

1 Department of Neurology, Mayo Clinic Arizona, Scottsdale, AZ, USA

2 Movement Disorders Center, Hackensack University Medical Center, Hackensack, NJ, USA a standing position or when getting out of bed. At that time, orthostatic blood pressure (BP) readings confirmed that she was experiencing a large fall in $\mathrm{BP}(170 / 87 \mathrm{mmHg}$ supine to $110 / 65 \mathrm{mmHg}$ after 3 -min of standing-up) while her heart rate (HR) increased by only 10 beats per minute or less; therefore, she was diagnosed with neurogenic orthostatic hypotension (nOH). Droxidopa was initiated at $100 \mathrm{mg}$ on a modified three times daily (TID) schedule (taken on awakening, at midday, and at least 3-4 h prior to bedtime) [1,3]. Her droxidopa dose was up-titrated every $24-48 \mathrm{~h}$ by $100 \mathrm{mg}$ on the modified TID schedule. She had a good symptomatic response with droxidopa at $500 \mathrm{mg}$ on the modified TID schedule and was experiencing much less frequent episodes of dizziness and lightheadedness, and no syncope.

\section{Expert commentary (Dr. Goodman)}

Prior to the initiation of droxidopa for $\mathrm{nOH}$ in this patient with MSA-P, it is important to identify goals of treatment and, ideally, to discuss these treatment goals with patients and their caregivers, when relevant. In patients with MSA, symptoms of $\mathrm{nOH}$ may include syncope, near syncope, lightheadedness when standing, fatigue, headache, or confusion. Recognition of these various symptoms provides not only the rationale for pursuing pharmacotherapy with droxidopa, but also will help establish treatment goals for individual patients.

\section{Case continuation}

The patient was on a stable dose of droxidopa $500 \mathrm{mg}$ on the modified TID schedule for approximately 6 months. However, she presented again with recurrent syncope, as well as other symptoms of nOH in a follow-up visit. 


\section{Expert commentary (Dr. Goodman)}

Syncope is a common manifestation of $\mathrm{nOH}$ in patients with MSA, and reducing the frequency of syncopal episodes is a major goal of treatment with droxidopa. A reduction in syncope in this patient for the initial 6 months following droxidopa initiation could be considered a treatment success. It is not uncommon for patients with $\mathrm{nOH}$ to have treatment reduce, but not fully abolish, nOH symptoms. Patients may also experience an exacerbation in symptoms during periods of prolonged bed rest or concomitant illness, including a recurrence of syncope. When evaluating a patient with worsening symptoms, including recurrent syncopal episodes, it is critical to rule out new medical problems (such as anemia or cardiac arrhythmia) or new medication that has resulted in new or recurrent symptoms. As such, a review of the patient's interval medical history and medications should be conducted. In addition, basic blood work and an electrocardiogram should also be ordered.

\section{Case continuation}

Review of medications, basic blood work, and electrocardiogram showed that were no new factors aggravating the fall in BP when standing. Thus, it was concluded that the likely cause of her recurrent syncope was progressing neurodegeneration, affecting autonomic pathways involved in BP control.

In this situation, for both patients and their caregivers, it is important to review again the non-pharmacologic measures to increase BP, including liberalization of salt and fluid intake, and wearing compression garments such as compression stockings or an abdominal binder. It is not uncommon for patients with MSA to forget some or all of these critically important lifestyle measures that are useful in enhancing the effectiveness of droxidopa. The patient and caregivers at the assisted living facility were instructed to make use of several of these lifestyle modifications; they were also instructed to ensure that the patient rested or slept with the head of the bed elevated by at least $30^{\circ}$ in order to avoid supine hypertension, a common complication in patients with MSA [2].

After reviewing again for potential medical and medication causes for new or worsening symptoms, adjustments in the patient's pharmacotherapeutic regimen should be considered. A mistake often made in situations such as this is to assume that droxidopa or other medications have failed and should be discontinued or substituted with an alternative medication.

In this patient with MSA-P, the cessation of syncope for
6 months after starting droxidopa indicated a significant treatment success, but she remained symptomatic. As the patient was not at the maximal dose of droxidopa, it was decided to up-titrate the patient to a higher dose of droxidopa, with the treatment goal of reducing or eliminating syncope. The patient was titrated up to the maximum dose of droxidopa at $600 \mathrm{mg}$ on the modified TID schedule. Unfortunately, even at the highest dosage of droxidopa, this patient continued to experience episodes of syncope. Her supine BP was $167 / 107 \mathrm{mmHg}$ with a HR of $71 \mathrm{bpm}$, which decreased to $95 / 59 \mathrm{mmHg}$ with a HR of $83 \mathrm{bpm}$ upon standing for $3 \mathrm{~min}$. In some circumstances, such as with this patient with MSA-P, despite maximal droxidopa doses, it may be necessary to add other medications for orthostatic hypotension.

Midodrine [6] is effective to treat $\mathrm{nOH}$ and syncope, starting at low dosages, and titrating upward with close monitoring. Sustained hypertension may be the limiting factor when combining droxidopa and midodrine, although no dedicated study has been specifically performed. Because syncope and other symptoms of $\mathrm{nOH}$ are typically worse in the mornings, higher doses of these medications might be used in the mornings, with smaller doses, or no adjunctive medications at all, administered in the afternoon or evening. For this patient, midodrine at $5 \mathrm{mg}$ on a modified BID schedule (taken on awakening and at midday) was added to her droxidopa regimen at $600 \mathrm{mg}$ TID. She was then titrated up to $10 \mathrm{mg}$ of midodrine on the modified BID schedule. While titrating midodrine, seated BP should be checked $1 \mathrm{~h}$ after each midodrine dose to ensure that the pressor effect is not too high. After reaching a steady dose of midodrine, she obtained adequate symptom control and was experiencing significantly fewer episodes of syncope, pre-syncope, dizziness, and lightheadedness. Her supine BP was $174 / 108 \mathrm{mmHg}$ with a HR of $68 \mathrm{bpm}$, which decreased to $110 / 89 \mathrm{mmHg}$ with a HR of $79 \mathrm{bpm}$ upon standing for $3 \mathrm{~min}$.

In addition to droxidopa and midodrine, other off-label medications could be considered in patients with MSA who are experiencing severe symptomatic $\mathrm{nOH}$. Pyridostigmine [5] is a medication that has been used in $\mathrm{nOH}$. It may be used in combination with droxidopa, although no dedicated study has been specifically performed. Desmopressin can be administered to patients with frequent nocturnal enuresis, which is common in MSA, and may help to decrease symptoms of $\mathrm{nOH}$ and syncope in the morning in these patients, although this has been shown in open-label small series $(<10$ patients) [4]. However, hyponatremia is a dangerous side effect of desmopressin. 


\section{Expert commentary (Dr. Gupta)}

MSA-P is one of the most challenging neurodegenerative disorders to treat. In addition to severe autonomic dysfunction, there are multiple motor and non-motor symptoms that need to be addressed. Levodopa can be used, typically with only minimal benefit, and may worsen $\mathrm{nOH}$. The degree of $\mathrm{nOH}$ in these patients is typically quite severe, and often a major cause of disability. Nonpharmacologic therapies are often ineffective alone; therefore, consideration of pharmacologic therapieswhether monotherapy or adjunctive therapy—is paramount. In this patient, droxidopa was a good choice based on the mechanism, efficacy, and tolerability; however, despite adequate dosing, this patient continued to suffer from syncopal episodes; therefore, consideration of adjunctive therapy, such as addition of midodrine, is appropriate. It is important to be vigilant about BP monitoring when adding additional pressor medications, particularly for hypertension, but in this case the benefit outweighed the risks.

\section{Compliance with ethical standards}

Conflict of interest Dr. Gupta is a consultant for Lundbeck. Dr. Goodman is a consultant for Lundbeck.

Funding source This manuscript is part of a supplement sponsored by Lundbeck.
Open Access This article is distributed under the terms of the Creative Commons Attribution 4.0 International License (http://creative commons.org/licenses/by/4.0/), which permits unrestricted use, distribution, and reproduction in any medium, provided you give appropriate credit to the original author(s) and the source, provide a link to the Creative Commons license, and indicate if changes were made.

\section{References}

1. Elgebaly A, Abdelazeim B, Mattar O, Gadelkarim M, Salah R, Negida A (2016) Meta-analysis of the safety and efficacy of droxidopa for neurogenic orthostatic hypotension. Clin Auton Res 26:171-180

2. Fanciulli A, Gobel G, Ndayisaba JP, Granata R, Duerr S, Strano S, Colosimo C, Poewe W, Pontieri FE, Wenning GK (2016) Supine hypertension in Parkinson's disease and multiple system atrophy. Clin Auton Res 26:97-105

3. Kaufmann H, Norcliffe-Kaufmann L, Palma JA (2015) Droxidopa in neurogenic orthostatic hypotension. Expert Rev Cardiovasc Ther 13:875-891

4. Mathias CJ, Fosbraey P, da Costa DF, Thornley A, Bannister R (1986) The effect of desmopressin on nocturnal polyuria, overnight weight loss, and morning postural hypotension in patients with autonomic failure. BMJ 293:353-354

5. Sandroni P, Opfer-Gehrking TL, Singer W, Low PA (2005) Pyridostigmine for treatment of neurogenic orthostatic hypotension [correction of hypertension] - a follow-up survey study. Clin Auton Res 15:51-53

6. Smith W, Wan H, Much D, Robinson AG, Martin P (2016) Clinical benefit of midodrine hydrochloride in symptomatic orthostatic hypotension: a phase 4, double-blind, placebo-controlled, randomized, tilt-table study. Clin Auton Res 26:269-277 